\title{
High Acceptance and Completion of HIV Self-testing Among Diverse Populations of Young People in Kenya Using a Community-Based Distribution Strategy
}

\author{
Kate S. Wilson ${ }^{1}$ (10 - Cyrus Mugo ${ }^{2}$ - David A. Katz ${ }^{1} \cdot$ Vivianne Manyeki $^{2} \cdot$ Carol Mungwala $^{2}$ - Lilian Otiso ${ }^{7}$. \\ David Bukusi $^{9} \cdot$ R. Scott McClelland ${ }^{1,5,6} \cdot$ Jane M. Simoni $^{1,4} \cdot$ Matt Driver $^{10} \cdot$ Sarah Masyuko $^{8} \cdot$ Irene Inwani $^{2}$. \\ Pamela K. Kohler ${ }^{1,3}$
}

Accepted: 26 August 2021 / Published online: 1 September 2021

(c) The Author(s), under exclusive licence to Springer Science+Business Media, LLC, part of Springer Nature 2021

\begin{abstract}
Oral HIV self-testing (HIVST) may expand access to testing among hard-to-reach reach adolescents and young adults (AYA). We evaluated community-based HIVST services for AYA in an urban settlement in Kenya. Peer-mobilizers recruited AYA ages 15-24 through homes, bars/clubs, and pharmacies. Participants were offered oral HIVST, optional assistance and post-test counseling. Outcomes were HIVST acceptance and completion (self-report and returned kits). Surveys were given at enrollment, post-testing, and 4 months. Log-binomial regression evaluated HIVST preferences by venue. Among 315 reached, $87 \%$ enrolled. HIVST acceptance was higher in bars/clubs (94\%) than homes (86\%) or pharmacies (75\%). HIVST completion was $97 \%$, with one confirmed positive result. Participants wanted future HIVST at multiple locations, include PrEP, and cost $\leq$ \$5USD. Participants from bars/clubs and pharmacies were more likely to prefer unassisted testing and peer-distributers compared to participants from homes. This differentiated community-based HIVST strategy could facilitate engagement in HIV testing and prevention among AYA.
\end{abstract}

Keywords HIV self-testing · Adolescents and young adults · Community-based testing $\cdot$ Differentiated HIV testing $\cdot$ SubSaharan Africa

\section{Introduction}

Adolescents and young adults (AYA) in sub-Saharan Africa (SSA) accounted for 33\% of all new infections in 2019 [1], and AIDS remains the leading cause of death in this population [2]. HIV testing is the entry point into prevention and treatment. However, HIV testing among AYA is lower

Kate S. Wilson

kateatheniawilson@gmail.com

1 Department of Global Health, Harborview Medical Center, University of Washington, 325 9th Avenue, Box 359931, Seattle, WA 98104, USA

2 University of Nairobi/Kenyatta National Hospital, Nairobi, Kenya

3 Department of Child, Family, and Population Health, Nursing, University of Washington, Seattle, USA

4 Department of Psychology, University of Washington, Seattle, USA than any other age group [3]. Common barriers for AYA to accessing provider-directed HIV testing services (HTS) include wait time, cost, stigma, and local consent laws [4-7].

Oral HIV self-testing (HIVST) is a convenient, safe, and accurate alternative to standard provider-directed HIV testing that can increase access and improve health system efficiencies, especially with service disruptions from 
COVID-19 [8-13]. Since the World Health Organization (WHO) recommended adopting HIVST in 2016, most countries in SSA have included HIVST into national HIV testing guidelines and differentiated service delivery strategies $[14,15]$. Community-based HIVST distribution is especially promising to increase testing and linkage to care and prevention among hard-to-reach populations $[16,17]$ and people who decline standard testing [18]. Secondary distribution through sexual partners or peers also improves routine testing among people with ongoing HIV exposure [19-21].

Recent studies show that HIVST is acceptable among AYA in SSA [7, 22]. In randomized trials of HIVST in Southern Africa, HIVST completion was higher among AYA than among older age groups [7, 23, 24]. However, there is limited evidence on how best to implement HIVST services to AYA in real-world settings $[25,26]$. It is also unclear whether AYA want independent self-testing options or provider-support models [27]. A study that included options of HIVST or provider-directed testing to support linkage to care among adolescent girls and young women (AGYW) in Kenya [28] found that a lower proportion of AGYW chose HIVST compared to provider-directed testing. Because AYA represent a heterogeneous population with diverse social contexts, preferences, and risk of HIV acquisition [29], it is important to evaluate multiple distribution points, including homes, pharmacies, and bars or bus stands, to reach distinct AYA where they are $[18,30]$. To address these gaps, we evaluated an HIVST model using three different communitybased distribution strategies on HIVST acceptance, completion, and preferences among AYA in Kenya.

\section{Methods}

\section{Setting and Population}

This cohort study was conducted in Kawangware, an informal settlement in Nairobi selected because it was a National AIDS and STD Control Programme priority area and had community-based HIV testing infrastructure and outreach programs for priority populations. Eligible AYA were ages 15-24 years, reported unknown or HIV-negative status, had access to a cell phone, and could provide written informed consent.

We purposefully selected three non-contiguous wards in Kawangware, one for each distribution channel: homebased testing (HBT), pharmacies, and 'hotspots' (bars/ nightclubs) hypothesized to reach different sub-groups of AYA. Recruitment varied by channel because of differences in when AYA were present. In HBT, we visited every fifth residence at different times and days during the week and approached all potentially eligible AYA present. Of 30 pharmacies visited, three were chosen based on $\geq 50$ customers per day, managers' willingness to refer clients to the study, and access to a private space. Pharmacy staff referred potentially eligible AYA customers to peer mobilizers. Of 20 hotspots where we had an established relationship, three were selected based on manager willingness to refer potential participants and provide a private space for study activities. Peer mobilizers approached all potentially eligible AYA and invited those interested to learn more about the study to a designated private room.

\section{Study Procedures}

Participants had three contacts with study staff: at enrollment, immediately after test completion, and at 4 months after enrollment. Peer mobilizers conducted recruitment and offered HIVST information, while HTS counselors performed study consent, screening, data collection, and HIVST services. At enrollment, participants completed an interviewer-administered survey in Kiswahili or English. Counselors provided pre-test counseling according to 2017 national self-testing guidelines [31], including an oral and video demonstration of how to take the self-test, interpret, and act on the results.

Each participant was offered one OraQuick In-Home HIV Test [32] with instructions in Kiswahili and English, a tollfree national hotline, and a referral card for retesting or linkage to care. All participants who accepted HIVST had the choice to test on their own or with assistance by study staff, according to national guidelines. Participants were requested to complete HIVST within 30 days and report the result by phone or text message. All participants were offered optional post-test counseling by phone or in-person from the study team or referral.

Participants who reported self-testing were contacted by study staff to return their used test and complete a user experience survey. Study staff verified HIVST results by reviewing returned test kits with participants. Participants with reactive or indeterminate HIVST results were offered point-of-care confirmatory blood-based rapid testing according to national guidelines. Participants with confirmed HIVpositive results were offered referral or escort to a preferred HIV care clinic. Participants with non-reactive results were advised according to national guidelines to retest at a local HTS facility in 3 months if they had a recent exposure to HIV, otherwise annually [31]. For quality assurance, confirmatory testing was performed on a $10 \%$ random sample of participants with non-reactive results. Four months after enrollment, participants completed a final survey in-person or by phone to assess linkage to care or retesting within 3 months and preferences for future HIVST distribution. We assessed linkage and retesting outcomes at 4 months after enrollment to allow time for participants to get a facilitybased HIV test. Participants who did not report their results 
and could not be reached by phone after three attempts were considered lost to follow-up.

\section{Outcomes}

Testing outcomes were HIVST acceptance (\% accepted HIVST, among eligible) and completion, defined as selftesting within 30 days among participants who accepted HIVST, by self-report. Returned used test kits were used to verify self-reports. Additional outcomes were: (1) test positivity (\% confirmed sero-positive results among AYA completing HIVST), (2) linkage to care among those confirmed to have HIV and (3) retesting at four months by self-report (\% retested among AYA who reported recent potential HIV exposure and had a non-reactive HIVST during the study. Potential recent exposure was determined by pre-exposure prophylaxis [PrEP] eligibility [33]. AYA who completed a HIVST were asked about their self-testing experiences, including choice of assistance and post-counseling, counseling by phone or in-person, ease of use (very vs. somewhat, not very, not at all), and disclosure of their test results to other people. We assessed intervention acceptability on the user experiences survey with the Acceptability of Intervention Measure, which consists of four statements with Likert scale options (e.g. HIVST meets my approval) [34]. At month four, we assessed future HIVST preferences about different venues (community spaces, facilities, online), modalities (in-person or phone/text), integration with other prevention services including PrEP, and willingness to pay (nothing, USD \$1-5, 6-9, 10-15). Among AYA who competed testing, intervention feasibility was assessed by recruitment efficiency (number of AYA who completed HIVST per day of recruitment) and proportions enrolled and retained [35].

\section{AYA Characteristics}

Enrollment and follow-up surveys assessed AYA characteristics and HIVST preferences guided by a socio-ecological framework of AYA health behavior [36]. Characteristics included age, education, partnership status, exposure to recent gender-based violence [37] (GBV; any act of physical, sexual, or emotional violence in the last 12 months by anyone, and by a romantic partner), ever had any vaginal or anal sex, any non-condom modern contraceptive use (hormonal pills or injectables, diaphragm, intrauterine device), condom use frequency (every time versus sometimes or never), history of sexually transmitted infections (STIs), transactional sex (exchange of sex for money or goods in the last 12 months), depressive symptoms by Patient Health Questionnaire-2 [38] (PHQ-2 score $\geq 3$ consistent with depression), potential alcohol use disorder using a 3-item Alcohol Use Disorders Identification Test-Consumption [39]
(AUDIT-C; score $\geq 4$ for males and $\geq 3$ for females, indicative of a potential disorder), any injection drug use, and HIV testing history. We defined PrEP eligibility according to Kenyan national guidelines, as reporting any sex with a partner of unknown or sero-positive status, injection drug use, sex with a partner who injects drugs, transactional sex, multiple sexual partners, forced sex, or STI symptoms in the last 6 months.

\section{Analyses}

We compared self-test acceptance, completion, retesting, and test experiences by channel using chi-square tests. Time to HIVST completion was compared by channels using Kaplan-Meier curves and log-rank tests, adjusted for age. We evaluated the association between recruitment channel and HIVST completion and retesting using univariable and multivariable log-binomial regression. We used logbinomial regression to evaluate pre-specified associations between recruitment channel and future testing preferences (any assistance or any post-test counseling by trained staff) and testing strategies under consideration in Kenya (peerdistributers, online, and vending machines). All final models were adjusted for age and HIV testing within 12 months prior to enrollment. Analyses were conducted in Stata 16.0 (College Station, Texas).

\section{Ethical Considerations}

This study was approved by the ethics committees at Kenyatta National Hospital (P35305/2018) and the University of Washington (STUDY00004396). All participants gave written informed consent. Study staff monitored for social harms using an assessment adapted from prior studies [23]. Any participant who indicated recent exposure to violence or had symptoms of depression by PHQ-2 or potential alcohol use disorder by AUDIT-C were offered referrals to local resources. Participants received \$3.00 reimbursement for their time for each visit.

\section{Results}

\section{Participant Enrollment}

Between November 16, 2018 and February 1, 2019, 342 individuals were approached for participation, of whom 322 were interested. Among these 322, 7 did not meet eligibility criteria for age $(n=4)$, HIV status $(n=2)$, and cell phone access $(n=1)$. An additional 38 declined enrollment (primary reasons: 'in a hurry', 'not willing', 'husband refused') and 3 were ineligible after revealing they were taking ART, for a final sample of 274 participants (87\% of eligible). 
Enrollment among 315 eligible AYA was highest in hotspots (94\%), followed by HBT (86\%) and pharmacies $(75 \%)$. Most were ages $18-24(75 \%)$, female $(64 \%)$, in a relationship (54\%) and reported HIV testing in the last 12 months (55\%) (Table 1). Participants had multiple vulnerabilities to HIV, especially those at hotspots and pharmacies. Overall, $48 \%$ of participants reported recent GBV, including $19 \%$ by a main partner and $24 \%$ by another person; $33 \%$ reported transactional sex in the last year; and 32\% had AUDIT-C scores consistent with alcohol use problems. Among 212 (77\%) AYA who reported ever having sex, 24\% reported using condoms every time, $27 \%(\mathrm{n}=184)$ using any noncondom modern contraceptives with a partner, and $19 \%$ $(n=199)$ reported STI symptoms in the last year. Nearly $58 \%$ were eligible for PrEP. Eligibility was most common among participants from hotspots $(88 \%)$, followed by pharmacies (57\%) then HBT (31\%).

\section{HIVST Acceptance, Completion, and Test Positivity}

All 274 participants accepted an HIVST, representing 87\% of all 315 eligible AYA approached for participation. This

Table 1 Participant characteristics by HIV self-test distribution channel

\begin{tabular}{|c|c|c|c|c|c|}
\hline Characteristic & $\begin{array}{l}\text { Home-based testing } \\
\mathrm{n}(\%) \text { or median IQR }(\mathrm{n}=121)\end{array}$ & $\begin{array}{l}\text { Pharmacies } \\
\mathrm{n}(\%) \text { or median IQR } \\
(\mathrm{n}=46)\end{array}$ & $\begin{array}{l}\text { Hot spots } \\
\mathrm{n}(\%) \text { or } \\
\text { median IQR } \\
(\mathrm{n}=107)\end{array}$ & $\begin{array}{l}\text { Total } \\
(\mathrm{N}=274)\end{array}$ & p-value, test statistics ${ }^{\mathrm{e}}$ \\
\hline \multicolumn{6}{|l|}{ Socio-demographics } \\
\hline Ages 18-24 years (vs 15-17) & $63(52.0)$ & $39(84.9)$ & $104(97.2)$ & $206(75.2)$ & $<0.001,64.7$ \\
\hline Female & $89(73.6)$ & $31(67.4)$ & $56(52.3)$ & $176(64.2)$ & $0.003,11.4$ \\
\hline In school & $73(60.3)$ & $12(26.1)$ & $13(12.2)$ & $89(35.8)$ & $<0.001,59.4$ \\
\hline In a relationship $(\mathrm{n}=271)$ & $48(40.3)$ & $28(60.9)$ & $73(68.9)$ & $149(54.4)$ & $<0.001,19.2$ \\
\hline \multicolumn{6}{|l|}{$\begin{array}{l}\text { Sexual behaviors and vulnerabili- } \\
\text { ties }\end{array}$} \\
\hline Ever had vaginal or anal sex & $64(54.2)$ & $42(91.3)$ & $106(99.1)$ & $212(77.4)$ & $<0.001,76.2$ \\
\hline $\begin{array}{l}\text { Use condoms 'every time' } \\
(\mathrm{n}=199)^{\mathrm{a}}\end{array}$ & $16(29.6)$ & $5(11.9)$ & $27(26.2)$ & $48(24.1)$ & $0.10,4.6$ \\
\hline $\begin{array}{l}\text { Any non-condom contraceptives } \\
(\mathrm{n}=184)^{\mathrm{b}}\end{array}$ & $11(22.0)$ & $15(35.7)$ & $32(34.8)$ & $58(31.5)$ & $0.10,10.7$ \\
\hline Eligible for $\operatorname{PrEP}(n=269)^{\mathrm{c}}$ & $38(31.4)$ & $26(56.5)$ & $94(87.9)$ & $158(57.7)$ & $<0.001,71.7$ \\
\hline $\begin{array}{l}\text { Any transactional sex, last } 12 \\
\text { months }^{\mathrm{a}}\end{array}$ & $5(4.1)$ & $4(9.7)$ & $80(74.8)$ & $89(32.5)$ & $<0.001,143.4$ \\
\hline $\begin{array}{l}\text { Physical, sexual or emotional vio- } \\
\text { lence, last } 12 \text { months }(\mathrm{n}=270)\end{array}$ & $47(38.8)$ & $15(32.6)$ & $67(62.6)$ & $129(47.8)$ & $<0.001,17.3$ \\
\hline $\begin{array}{l}\text { Any violence by a romantic part- } \\
\text { ner }(n=124)^{d}\end{array}$ & $8(17.0)$ & $6(16.2)$ & $10(25.0)$ & $24(19.1)$ & $0.54,1.2$ \\
\hline $\begin{array}{l}\text { Any STI symptoms, last } \\
12 \text { months }(\mathrm{n}=199)\end{array}$ & $7(12.5)$ & $5(11.9)$ & $26(25.7)$ & $38(19.1)$ & $0.05,5.9$ \\
\hline $\begin{array}{l}\text { Potential alcohol use disorder } \\
\quad(\text { AUDIT-C } \geq 3)\end{array}$ & $7(5.8)$ & $7(15.2)$ & $73(68.2)$ & $87(31.8)$ & $<0.001,109.1$ \\
\hline $\begin{array}{l}\text { Depressive symptoms } \\
(\text { PHQ-2 } \geq 3)(\mathrm{n}=269)\end{array}$ & $13(10.8)$ & $5(10.9)$ & $18(17.5)$ & $36(13.4)$ & $0.30,2.4$ \\
\hline \multicolumn{6}{|l|}{ HIV test history } \\
\hline $\begin{array}{l}\text { Tested for HIV in the last } \\
12 \text { months }\end{array}$ & $56(46.3)$ & 27 (58.7) & $67(62.2)$ & $150(54.7)$ & $0.04,6.5$ \\
\hline Ever taken an HIVST & $2(1.7)$ & $0(0)$ & $10(9.4)$ & $12(4.4)$ & - \\
\hline
\end{tabular}

PHQ-2 Patient Health Questionnaire-2; AUDIT-C Alcohol Use Disorders Identification Test-Consumption; HIVST oral HIV self-test; PrEP preexposure prophylaxis; STI sexually transmitted infection

${ }^{a}$ Among participants who reported ever having sex;

${ }^{\mathrm{b}}$ Non-condom modern methods were hormone injections, implants, pills, or intrauterine device;

${ }^{\mathrm{c}}$ PrEP eligibility defined as reporting yes to any of the following in the last the 6 months: sex with a partner of unknown or sero-positive status, any injection drug use, sex with a partner who injects drugs, any transactional sex, multiple sexual partners, forced sex, or STI symptoms

${ }^{\mathrm{d}}$ Among participants who reported having a spouse or girlfriend/boyfriend

e Pearson's Chi-square tests 
percentage was higher in hotspots (94\%) than HBT $(86 \%)$ or pharmacies (75\%) (Table 2). Self-reported HIVST completion was $97 \%$, with no significant differences by channel, gender or age. Median time to testing was one day (interquartile range [IQR] $0.5-3$ days), with minimal differences in days between hotspots ( 0.5 days, IQR $0.5-1)$, pharmacy ( 1 day, IQR $0.5-3$ ) and HBT ( 2 days, IQR $0.5-3, \chi^{2}=29.0$, log rank p-value $<0.001)$. Among 266 participants who tested, $256(96 \%)$ returned their test kit for verification. Ten participants (3\%) reported reactive HIVST results and returned their test kits. Of these 10 kits, study staff verified that four were reactive and six were non-reactive. Study staff reviewed the test kit results with the six participants who had reported reactive results and determined that the discrepancy was due to participants' confusion in reading the test strip correctly. The four participants with reactive results received confirmatory blood-based rapid testing. One test was confirmed positive and three confirmed negative (i.e. three false positives). Overall test positivity was $0.4 \%$. The $10 \%$ random sample $(n=26)$ of non-reactive results tested for quality assurance were all confirmed negative. No social harms or unanticipated events related to the study were reported.

\section{Feasibility of HIVST Model}

The team recruited 315 eligible AYA in 60 days. Recruitment efficiency was highest in hotspots, with 11 test completions per recruitment day versus 4 per day in HBT, and 2 per day in pharmacies. Retention at 4 months was $94 \%$, with no differences by channel. Of 17 participants not retained, 11 (4\%) were lost to follow-up and $6(2 \%)$ withdrew because they had left Nairobi.

\section{HIVST Experiences and Acceptability}

Of 266 participants with self-test results, $95 \%$ completed the testing experience surveys. Overall, $92 \%$ self-tested without assistance from study staff, with no significant differences by age (ages 15-17: 63, 94\%; ages 18-24, 183: 92\%, $\mathrm{p}=0.41$, $\chi^{2}=0.67$ ) or gender (female $160,93 \%$ vs. male $86,91 \%$, $\left.\mathrm{p}=0.40, \chi^{2}=0.73\right)$. Most participants $(57 \%)$ disclosed their HIVST results to someone outside the study, primarily a caregiver (68\%), friend (63\%), or partner (36\%) (Table 3). Nearly half (48\%) received post-test counseling, of which 93\% was in-person. Most (83\% of 253) reported that the HIVST was very easy to use, and $87 \%$ reported being very confident interpreting results by themselves, although $15 \%$ reported difficulty with at least one step. Based on the four questions of the Acceptability Intervention Measure, acceptability of HIVST services was high among AYA, with $\geq 90 \%$ reporting that they somewhat or completely agreed that HIVST met their approval, was pleasing, was welcomed, and was liked. Seventy-nine percent would take an HIVST at their next test instead of a provider-delivered test $(11 \%$ were unsure) because it was easier to use (100\%), private (99\%), and gave trustworthy results (91\%). The $16 \%$ who preferred

Table 2 Participant characteristics by HIV self-test distribution channel

\begin{tabular}{|c|c|c|c|c|c|}
\hline Oral HIV self- testing outcomes & $\begin{array}{l}\text { Home-based } \\
\text { testing } \\
\mathrm{n}(\%) \text { or median } \\
\text { IQR }(\mathrm{n}=121)\end{array}$ & $\begin{array}{l}\text { Pharmacies } \\
\mathrm{n}(\%) \text { or median IQR } \\
(\mathrm{n}=46)\end{array}$ & $\begin{array}{l}\text { Hot spots } \\
\mathrm{n}(\%) \text { or median } \\
\text { IQR }(\mathrm{n}=107)\end{array}$ & Total $(\mathrm{N}=274)$ & $\begin{array}{l}\text { p-value, test statis- } \\
\text { tics on pre-specified } \\
\text { comparisons }{ }^{\mathrm{e}}\end{array}$ \\
\hline HIVST acceptance at enrollment & $121 / 142(85.8)$ & 46/61 (75.4) & $107 / 112(93.8)$ & $274(87.2)^{\mathrm{a}}$ & $<0.001,14.8$ \\
\hline Test completion by self-report or used kit & $118(97.5)$ & $43(93.5)$ & $105(98.1)$ & $266(97.1)$ & $0.27,2.61$ \\
\hline HIVST results & $56(46.3)$ & $26(56.5)$ & $81(75.7)$ & $163(61.3)$ & \\
\hline Reactive by self-report & 2 & 1 & 4 & $7^{\mathrm{b}}$ & - \\
\hline Non-reactive by self-report & 116 & 45 & 101 & 259 & - \\
\hline Confirmed sero-positive & 0 & 1 & 0 & 1 & - \\
\hline Confirmed sero-negative $^{c}$ & 118 & 45 & 105 & 265 & - \\
\hline $\begin{array}{l}\text { Self-reported retesting on own by } 4 \text { months } \\
(\mathrm{n}=265)\end{array}$ & $28(24.1)$ & $15(35.7)$ & $53(54.1)$ & $96(37.5)$ & $<0.001,20.4$ \\
\hline Among prep eligible $(n=116)^{\mathrm{d}}$ & $4(22.0)$ & $5(45.5)$ & $47(54.2)$ & $56(48.3)$ & $0.05,6.08$ \\
\hline
\end{tabular}

HIVST oral HIV self-test

${ }^{a}$ Among 315 AYA eligible for a HIVST

${ }^{\mathrm{b}}$ Three self-reported reactive results were verified as non-reactive by study staff when they received the used kits. These discrepancies were due to participant error interpreting the test result

${ }^{\mathrm{c}}$ Among 265 participants who completed the HIVST and had non-reactive results

${ }^{\mathrm{d}}$ PrEP eligibility defined as reporting yes to any of the following in the last the 6 months: sex with a partner of unknown or sero-positive status, any injection drug use, sex with a partner who injects drugs, any transactional sex, multiple sexual partners, forced sex, or STI symptoms [33]

${ }^{\mathrm{e}} \mathrm{Chi}$-squared test statistics were run only on pre-specified comparisons by channel 
Table 3 User experiences and future testing strategies by distribution channel

\begin{tabular}{|c|c|c|c|c|c|}
\hline Oral HIV self- testing outcomes & $\begin{array}{l}\text { Home-based } \\
\text { testing } \\
\mathrm{n}(\%) \text { or median } \\
\text { IQR }(\mathrm{n}=118)\end{array}$ & $\begin{array}{l}\text { Pharmacies } \mathrm{n}(\%) \text { or } \\
\text { median IQR }(\mathrm{n}=43)\end{array}$ & $\begin{array}{l}\text { Hot spots } \\
\mathrm{n}(\%) \text { or median } \\
\text { IQR }(\mathrm{n}=105)\end{array}$ & Total $(\mathrm{N}=266)$ & $\begin{array}{l}\text { p-value, test statis- } \\
\text { tics on pre-specified } \\
\text { comparisons }^{\mathrm{a}}\end{array}$ \\
\hline \multicolumn{6}{|l|}{ Self-testing and post-testing steps } \\
\hline Assisted by study staff & $5(4.2)$ & $3(7.0)$ & $12(11.4)$ & $20(7.5)$ & $0.13,4.2$ \\
\hline Any posttest counseling by study staff & $59(49.2)$ & $20(43.5)$ & $51(48.6)$ & $130(48.0)$ & - \\
\hline In-person (vs phone) & $56(94.9)$ & $19(95.0)$ & $45(88.2)$ & $120(93.0)$ & - \\
\hline Non-study staff present when testing & $30(28.0)$ & $7(16.7)$ & $17(16.2)$ & $54(21.3)$ & - \\
\hline Disclosed result to someone & $75(70.1)$ & $22(52.4)$ & $48(46.2)$ & $145(57.1)$ & $0.002,12.9$ \\
\hline Caregiver & $58(77.3)$ & $14(63.6)$ & $27(56.8)$ & $99(68.3)$ & - \\
\hline Friend & $40(53.3)$ & $12(54.6)$ & $39(83.0)$ & $91(63.2)$ & - \\
\hline Sexual partner & $35(34.3)$ & $14(33.3)$ & $40(38.8)$ & $89(36.0)$ & - \\
\hline \multicolumn{6}{|l|}{ Usability and acceptability of oral HIVST } \\
\hline Very easy to use overall $(n=253)$ & $84(78.5)$ & $36(85.7)$ & $90(86.5)$ & $210(83.0)$ & - \\
\hline Very easy to see pink line & $90(84.1)$ & $32(76.2)$ & $91(88.4)$ & $213(85.2)$ & - \\
\hline Very easy to collect saliva & $90(84.1)$ & $35(83.3)$ & $90(85.7)$ & $215(84.7)$ & - \\
\hline Very easy to run test & $80(74.8)$ & $34(81.0)$ & $93(88.6)$ & $207(81.5)$ & - \\
\hline Very easy to interpret results & $90(84.1)$ & $35(83.3)$ & $95(90.5)$ & $220(86.6)$ & - \\
\hline Very confident interpreting results & $95(88.8)$ & $35(85.4)$ & $89(84.8)$ & $219(86.6)$ & - \\
\hline Trusted results of the test & $90(84.9)$ & $33(80.5)$ & $94(89.5)$ & $217(86.1)$ & - \\
\hline \multicolumn{6}{|l|}{ Preferred test type for next test } \\
\hline Oral HIVST & $77(74.6)$ & $34(82.9)$ & $78(83.0)$ & $189(79.4)$ & - \\
\hline Provider-delivered testing & $23(19.5)$ & $7(16.3)$ & $13(12.4)$ & $43(16.2)$ & $0.30,2.4$ \\
\hline Unsure & $15(12.7)$ & $2(4.7)$ & $11(10.5)$ & $28(10.5)$ & - \\
\hline $\begin{array}{l}\text { Would recommend HIVST to a friend } \\
(\mathrm{n}=252)\end{array}$ & $103(96.3)$ & $42(100)$ & $104(99.1)$ & $249(98.8)$ & - \\
\hline \multicolumn{6}{|l|}{ Acceptability of Intervention Measure [34] ${ }^{\mathrm{b}}$} \\
\hline I welcome HIVST services & $103(96.3)$ & $37(88.1)$ & $100(96.2)$ & $240(94.9)$ & - \\
\hline HIVST meets my approval & $96(92.3)$ & $36(85.7)$ & $93(89.4)$ & $225(90.0)$ & - \\
\hline I like HIVST & $96(92.3)$ & $35(83.3)$ & $97(92.4)$ & $228(90.5)$ & - \\
\hline HIVST is appealing & $101(94.4)$ & $37(88.1)$ & $100(95.2)$ & $238(93.7)$ & - \\
\hline
\end{tabular}

HIVST oral HIV self-test

${ }^{\mathrm{a}} \mathrm{Chi}$-squared test statistics were run on pre-specified comparisons by channel

besponse options ranged were 'completely disagree,' 'somewhat disagree,' ‘somewhat agree,' and 'completely agree,' which were collapsed into agree/disagree in this analysis

provider-delivered testing reported it was more accurate (95\%), trustworthy (93\%), and used by friends (77\%).

\section{Retesting by 4 Months}

Overall, 38\% of participants reported retesting at an HTS site. This percentage was higher among participants from hotspots (54\%) and pharmacies (36\%) compared to HBT $\left(24 \%, \mathrm{p}<0.001, \chi^{2}=20.4\right)$. Retesting was more common among participants eligible for PrEP compared to those who were not $\left(116,48 \%\right.$ vs. $\left.38,28 \%, p=0.001, \chi^{2}=11.9\right)$. The main reasons for not retesting by four months were lack of time and intent to retest within 12 months.

\section{Future HIVST Preferences}

The most common strategies that participants recommended to raise awareness about HIVST among AYA were through flyers (86\%), social media (75\%), TV and radio (64\%), and peer educators (51\%). Overall, $31 \%$ wanted HIVST assistance by a trained provider or peer educator, and $75 \%$ wanted post-test counseling, preferably in-person (89\%) (Table 4). In regression modeling adjusted for age and prior HIV testing, preference for no assistance was significantly higher among participants from hotspots and pharmacies compared to HBT (adjusted risk ratio [aRR] pharmacy: 1.43, 95\% CI 1.15-1.77; aRR hotspots: $1.19,95 \%$ CI $0.95-1.48$, Global 
Table 4 Preferences for future HIVST venues and models among AYA at month-4 follow-up $(\mathrm{N}=257)$

HBT $(n=116) \quad$ Pharmacy $(n=42) \quad$ Hotspot $(n=99) \quad$ Total $(n=257) \quad$ p-value, test statistics on pre-specified comparisons $^{\mathrm{a}}$

\begin{tabular}{|c|c|c|c|c|c|}
\hline \multicolumn{6}{|l|}{ HIVST distribution venue/mode } \\
\hline \multicolumn{6}{|l|}{ Study venues } \\
\hline HBT & $63(54.8)$ & $15(35.7)$ & $19(19.4)$ & $97(38.0)$ & - \\
\hline Pharmacy & $48(41.7)$ & $26(61.9)$ & $15(15.3)$ & $89(34.9)$ & - \\
\hline Hotspots & $0(0)$ & $0(0)$ & $60(61.2)$ & $60(23.5)$ & - \\
\hline \multicolumn{6}{|l|}{ Other venues } \\
\hline Sports event & $29(25.0)$ & $12(28.6)$ & $28(28.3)$ & $69(26.9)$ & - \\
\hline Youth-friendly event or center & $56(48.3)$ & $18(42.9)$ & $44(44.4)$ & $118(45.9)$ & - \\
\hline Health facility & $63(54.3)$ & $23(54.8)$ & $62(62.6)$ & $148(57.6)$ & - \\
\hline Church & $12(10.3)$ & $2(4.8)$ & $3(3.0)$ & $17(6.6)$ & - \\
\hline Online & $24(20.7)$ & $15(35.7)$ & $17(17.2)$ & $56(21.8)$ & $0.05,6.1$ \\
\hline Vending machine & $12(10.3)$ & $12(28.6)$ & $13(13.1)$ & $37(14.4)$ & $0.01,8.5$ \\
\hline \multicolumn{6}{|l|}{ Interpersonal distribution } \\
\hline Friend & $1(0.9)$ & $1(2.4)$ & $4(4.0)$ & $6(2.3)$ & - \\
\hline Trained peer educator & $34(29.3)$ & $13(31.0)$ & $46(46.5)$ & $93(36.2)$ & $0.03,7.4$ \\
\hline Sexual partner & $10(8.6)$ & $2(4.8)$ & $14(14.1)$ & $26(10.1)$ & - \\
\hline Chose $\geq 2$ of above options & $116(100.0)$ & $42(100.0)$ & 99 (100.0) & $257(100.0)$ & - \\
\hline \multicolumn{6}{|l|}{ Types of support with HIVST } \\
\hline Testing assistance & $44(38.3)$ & $6(14.3)$ & $28(28.6)$ & $78(30.6)$ & $0.01,8.6$ \\
\hline Post-test counseling & $87(76.3)$ & $26(61.9)$ & $78(79.6)$ & $191(75.2)$ & $0.08,5.1$ \\
\hline \multicolumn{6}{|l|}{ Mode of counseling $(n=191)$} \\
\hline In-person & $76(87.4)$ & $23(88.5)$ & $71(91.0)$ & $170(89.1)$ & - \\
\hline Phone or text messaging & $8(9.2)$ & $3(11.5)$ & $6(7.7)$ & $17(8.9)$ & - \\
\hline Using social media & $3(3.5)$ & $0(0)$ & $1(1.3)$ & $4(2.1)$ & - \\
\hline
\end{tabular}

HIVST oral HIV self-test, $H B T$ home-based testing

${ }^{a}$ Chi-squared test statistics were run on pre-specified comparisons by channel

p-value $\left.=0.005, \chi^{2}=10.6\right)$. All participants wanted at least two different venues to access HIVST. A significantly higher proportion of participants from pharmacies and hotspots compared to HBT would get HIVST through vending machines (aRR pharmacy: 2.58 , 95\% CI 1.09-6.10; hotspots: 1.17, 95\% CI 0.47-2.90, Global p-value $\left.=0.03 \chi^{2}=7.19\right)$ and peer-distributors (aRR pharmacy: $1.09,95 \%$ CI $0.62-1.91$; aRR hotspots: $1.65,95 \%$ CI 1.08-2.52, Global p-value $=0.05 \chi^{2}=6.07$ ). Overall, 96\% said they would distribute HIVST kits to a median of 2 sexual partners (IQR 1-4) and 10 friends (IQR 5-20) each. The majority (63\%) would not pay anything for a HIVST, while $36 \%$ would pay up to $\$ 5$ ( $1 \%$ would pay $\$ 6-10$ ), with no significant differences by age or gender. When asked what additional services to include with HIVST, most requested partner notification (90\%), STI screening (88\%), GBV counseling (88\%), contraceptives (85\%), and PrEP (79\%).

\section{Discussion}

This differentiated community-based strategy to offer oral HIVST to AYA in Kenya demonstrated high levels of selftesting acceptance and completion. Recruitment of AYA through HBT, pharmacies, and hotspots reached AYA with different HIV risk factors and self-testing preferences. Participants at hotspots reported the highest levels of violence and preferences for unassisted testing and peer-distributed testing models. Overall, participants wanted different community-based delivery self-testing options and different places to obtain HIVST.

In this study, HIVST acceptance was $87 \%$, and completion was $97 \%$, higher than other studies in sub-Saharan Africa [7, 18, 28, 40]. In a study among AGYW in Western Kenya where participants were offered a choice between HIVST and provider-delivered testing, 22\% chose 
self-testing compared to $78 \%$ provider testing [28]. While we did not directly evaluate a choice between HIVST and a standard provider-delivered rapid testing, $73 \%$ of participants said they would select self-testing next time they tested while $16 \%$ would choose provider-delivered testing. A community-randomized trial in Swaziland where household members distributed HIVST kits to AYA ages 12-24 who had been absent for standard HBT reported that $75 \%$ of AYA completed HIVST in the intervention communities compared to $39 \%$ of AYA in control communities who were offered a referral to test at a facility [18]. Differing results across studies may be due to differences in test kit distribution models and whether choices were offered [19, 28]. Our study engaged local youth peer mobilizers and HTS counselors as study staff. Peer educators can improve intervention uptake because they are familiar, trustworthy, and relatable, especially for hard-to-reach populations [17, 19]. This may help explain the particularly high enrollment and retention among AYA at hotspots. Our study extends prior findings by demonstrating high acceptance and completion of HIVST across diverse sub-groups of AYA at different venues.

Recruiting participants through three distinct channels was a feasible strategy to engage sub-populations AYA with different risks and service needs. Recruitment efficiency was highest in hotspots and lowest in pharmacies. This may be because hotspots are familiar sites for offering other HIV testing and prevention services and may be more comfortable sites for AYA to test. In contrast, pharmacies were less known for offering HIV services. A higher proportion of participants from hotspots compared to the other channels had tested before, were male, and were eligible for PrEP. They also experienced higher levels of GBV and alcohol use $[28,29]$. Tailored HIVST services at hotspots could include brief empowerment and harm reduction interventions [41]. In contrast, participants from HBT tended to be younger, female, with lower reported risk behaviors, and had someone present when they tested. Home-based self-testing for AYA could be an entry into offering family-centered prevention and care services [42]. The pharmacy channel reached an older, mixed gender population with lower reported risk behaviors than AYA from hotspots. Pharmacists could be trained to offer youth-tailored HIVST services and to distribute test kits and other HIV prevention products, including PrEP [43].

Most participants reported positive self-testing experiences and wanted future HIVST models to include a variety of service options, from fully independent to fully supported models. As in other studies, most participants said test kits were easy to use, convenient, private, and trustworthy, and would self-test again $[19,44]$. Over $90 \%$ of participants completed the HIVST without assistance regardless of age. However, the majority wanted their next self-testing experience to include the option to receive support during self-testing and post-test counseling. Studies that included AYA in sub-Saharan Africa [28, 40, 45] have reported preferences for provider-supported self-testing, given that some people report concerns about reading the test correctly and getting follow-up care. This highlights the importance of differentiated HTS for AYA tailored to individuals and contexts, where different testing products, venues, and services packages are offered to maximize access to HTS [8].

Notably, a peer-distribution model of HIVST was particularly popular among hotspot participants. Studies with adult women in sex work in Zambia and Uganda [46] and MSM in South Africa [17] and the US [47] have shown that these models are effective at increasing HIV testing completion among priority populations and should be evaluated with AYA. Task-shifting of additional HIVST and prevention services to lay cadres could enhance efficiency of HTS [48-50]. Consistent with studies among adults in sub-Saharan Africa, we found that willingness to pay for HIVST was low, and most AYA wanted HIVST for free $[5,25,51]$. HIVST offered through pharmacies, vending, machines or online will require fair pricing so that these strategies for increasing access by location are not limited by cost. Given widespread use of cell phones and social media among AYA in sub-Saharan Africa [48, 52], digital platforms have the potential to increase demand for HIVST among AYA and complement in-person counseling through live chats with trained counselors. Finally, our study reached young people with high unmet needs for additional HIV/STI and sexual and reproductive health (SRH) services. Greater integration of HIV and SRH services is recommended for youth in resource-limited settings [53]. HIVST can facilitate PrEP continuation [11] and ART initiation [54]. This flexible, community-based delivery model for HIVST could be expanded to offer a combination of client-centered SRH services for this priority population.

Test positivity was lower than reported in other HIVST studies in sub-Saharan Africa, likely due to lower background HIV prevalence and higher proportion of participants who had tested within 12 months [7, 23]. Notably, our study was not designed to evaluate HIVST for case finding, which may require a more targeted sampling strategy or larger sample size $[20,55,56]$. Participants with lower HIV risk may self-select into HIVST studies than individuals who do not participate [7]. We also identified a few AYA with false positive HIVST results, which were due to confusion with test instructions [57-59] or interpreting results after the recommended 40 min $[60,61]$. This highlights the importance of further refining standard HIVST instructions with AYA to minimize user errors and unnecessary distress.

Nearly $50 \%$ of AYA who were eligible for PrEP also reported retesting within four months, and this was more common among participants recruited from hotspots and 
pharmacies. This suggests many of these participants can access routine testing with minimal support, although they may benefit from receiving multiple HIVST kits to facilitate retesting. Studies designed to increase access to HIVST by offering multiple HIVST kits to participants reported higher rates of retesting [17, 47]. Community-based models using lay health workers could be evaluated to distribute HIVST to support uptake of PrEP or ART among AYA.

Strengths of this study include the evaluation of three community-based distribution channels, which enhanced generalizability to other settings, recruitment of diverse populations of AYA, engagement of local lay-health workers as study staff, and collection of implementation outcomes. We evaluated HIVST completion using both self-report and used tests, which adds methodologic rigor. Limitations included the observational design without a comparison group, which may have overestimated test acceptance and completion since AYA were not offered another option [28], non-random selection of recruitment channels, and self-selection of potentially lower risk AYA. Self-reported measures were subject to social desirability and recall bias. Due to low test positivity, we could not evaluate differences in test positivity by recruitment channel. Participants received reimbursements and on-demand access to study staff, which may be lower in real-world HIVST programs with AYA.

\section{Conclusion}

In summary, we found that a differentiated community-based HIVST model using lay heath workers was feasible in reaching a diverse population of AYA at risk for HIV/STI and unintended pregnancy. This model is a promising platform to improve HIV testing coverage efficiently, engage AYA with additional client-led prevention and care services, and help countries achieve the 2030 targets for HIV elimination among young people.

Acknowledgements We thank all participants in this study, LVCTHealth for collaboration and access to community stakeholders and study venues, Kenyatta National Hospital and the Kenya National AIDS and STD Control Program for collaboration and procurement of HIVST kits, University of Washington (UW) and University of Nairobi study teams, and the SPEED Study adolescent community advisory board. We thank the UW/Fred Hutch Center for AIDS Research program for supporting this study.

Author contributions KW, II, CM, LO, SM, DK, PK, RSM, JS, and DB designed the study, VM, CM, and LO collected data, II and LO oversaw field activities, SM and DB procured HIVST kits, MD managed the data, KW conducted the analysis, $\mathrm{KW}$ drafted the manuscript, and all authors reviewed, edited, and approved the final manuscript.

Funding This research was funded by a 2017 developmental grant to KW from the University of Washington / Fred Hutch Center for AIDS Research, an NIH funded program under award number AI027757 which is supported by the following NIH Institutes and Centers: NIAID, NCI, NIMH, NIDA, NICHD, NHLBI, NIA, NIGMS, NIDDK.

Data availability De-identified study related data will be made available on request.

\section{Declarations}

Conflict of interest The authors have no financial conflicts of interest to declare.

Ethical approval This study was approved by ethics committees at Kenyatta National Hospital (P35305/2018) and University of Washington (STUDY00004396).

Consent to Participate All participants gave written informed consent.

Consent for Publication The authors consent to publish.

\section{References}

1. UNAIDS. Global AIDS update 2020: Seizing the moment; Tackling entrenched inequalities to end epidemics Geneva, Switzerland; 2020. https://www.unaids.org/sites/default/files/media_asset/ 2020_global-aids-report_en.pdf.

2. UNAIDS. Global AIDS Monitoring 2020. 2019 https://www. unaids.org/sites/default/files/media_asset/global-aids-monitoring_ en.pdf.

3. AVERT. Global information and education on HIV and AIDS: Young people, HIV and AIDS 2020. https://www.avert.org/profe ssionals/hiv-social-issues/key-affected-populations/young-people.

4. Strauss M, Rhodes B, George G. A qualitative analysis of the barriers and facilitators of HIV counselling and testing perceived by adolescents in South Africa. BMC Health Serv Res. 2015;15:250

5. Strauss M, George G, Rhodes B. Designing human immunodeficiency virus counselling and testing services to maximize uptake among high school learners in South Africa: what matters? Sex Transm Dis. 2017;44(5):290-6.

6. McKinnon B, Vandermorris A. National age-of-consent laws and adolescent HIV testing in sub-Saharan Africa: a propensity-score matched study. Bull World Health Organ. 2019;97(1):42-50.

7. Hatzold K, Gudukeya S, Mutseta MN, Chilongosi R, Nalubamba M, Nkhoma C, et al. HIV self-testing: breaking the barriers to uptake of testing among men and adolescents in sub-Saharan Africa, experiences from STAR demonstration projects in Malawi, Zambia and Zimbabwe. J Int AIDS Soc. 2019;22:e25244.

8. World Health Organization. HIV self-testing and partner notification: supplement to consolidated guidelines on HIV testing services 2016. https://apps.who.int/iris/bitstream/handle/10665/ 251655/9789241549868-eng.pdf?sequence $=1$.

9. Pant Pai N, Balram B, Shivkumar S, Martinez-Cajas JL, Claessens C, Lambert G, et al. Head-to-head comparison of accuracy of a rapid point-of-care HIV test with oral versus whole-blood specimens: a systematic review and meta-analysis. Lancet Infect Dis. 2012;12(5):373-80

10. Pant Pai N, Sharma J, Shivkumar S, Pillay S, Vadnais C, Joseph $\mathrm{L}$, et al. Supervised and unsupervised self-testing for HIV in high- and low-risk populations: a systematic review. PLoS Med. 2013;10(4):e1001414.

11. Ortblad KF, Kearney JE, Mugwanya K, Irungu EM, Haberer JE, Barnabas RV, et al. HIV-1 self-testing to improve the efficiency of 
pre-exposure prophylaxis delivery: a randomized trial in Kenya. Trials. 2019;20(1):396.

12. Mhango M, Chitungo I, Dzinamarira T. COVID-19 lockdowns: impact on facility-based HIV testing and the case for the scaling up of home-based testing services in sub-Saharan Africa. AIDS Behav. 2020;24(11):3014-6.

13. Johnson CC, Kennedy C, Fonner V, Siegfried N, Figueroa C, Dalal $\mathrm{S}$, et al. Examining the effects of HIV self-testing compared to standard HIV testing services: a systematic review and metaanalysis. J Int AIDS Soc. 2017;20(1):1-10.

14. Tonen-Wolyec S, Mbopi-Kéou FX, Koyalta D, Filali M, BatinaAgasa S, Bélec L. Human immunodeficiency virus self-testing in adolescents living in Sub-Saharan Africa: an advocacy. Niger Med J. 2019;60(4):165-8.

15. Tonen-Wolyec S, Koyalta D, Mboumba Bouassa RS, Filali M, Batina-Agasa S, Bélec L. HIV self-testing in adolescents living in Sub-Saharan Africa. Med Mal Infect. 2020;50:648.

16. Ortblad K, Kibuuka Musoke D, Ngabirano T, Nakitende A, Magoola J, Kayiira P, et al. Direct provision versus facility collection of HIV self-tests among female sex workers in Uganda: a cluster-randomized controlled health systems trial. PLoS Med. 2017;14(11):e1002458.

17. Lippman SA, Lane T, Rabede O, Gilmore H, Chen YH, Mlotshwa $\mathrm{N}$, et al. High acceptability and increased HIV-testing frequency after introduction of HIV self-testing and network distribution among South African MSM. J Acquir Immune Defic Syndr. 2018;77(3):279-87.

18. Amstutz A, Lejone TI, Khesa L, Muhairwe J, Bresser M, Vanobberghen F, et al. Home-based oral self-testing for absent and declining individuals during a door-to-door HIV testing campaign in rural Lesotho (HOSENG): a cluster-randomised trial. Lancet HIV. 2020;7(11):e752-61.

19. Choko AT, Nanfuka M, Birungi J, Taasi G, Kisembo P, Helleringer S. A pilot trial of the peer-based distribution of HIV self-test kits among fishermen in Bulisa, Uganda. PLoS ONE. 2018;13(11):e0208191.

20. Thirumurthy H, Masters SH, Mavedzenge SN, Maman S, Omanga E, Agot K. Promoting male partner HIV testing and safer sexual decision making through secondary distribution of self-tests by HIV-negative female sex workers and women receiving antenatal and post-partum care in Kenya: a cohort study. Lancet HIV. 2016;3(6):e266-74.

21. Pintye J, Drake AL, Begnel E, Kinuthia J, Abuna F, Lagat H, et al. Acceptability and outcomes of distributing HIV self-tests for male partner testing in Kenyan maternal and child health and family planning clinics. AIDS. 2019;33(8):1369-78.

22. Tonen-Wolyec S, Mbopi-Keou FX, Batina-Agasa S, Kalla GCM, Noubom M, Mboumba Bouassa RS, et al. Acceptability of HIV self-testing in African students: a cross-sectional survey in the Democratic Republic of Congo. Pan Afr Med J. 2019;33:83.

23. Choko AT, MacPherson P, Webb EL, Willey BA, Feasy H, Sambakunsi R, et al. Uptake, accuracy, safety, and linkage into care over two years of promoting annual self-testing for HIV in Blantyre, Malawi: a community-based prospective study. PLoS Med. 2015;12(9):e1001873.

24. Mulubwa C, Hensen B, Phiri MM, Shanaube K, Schaap AJ, Floyd S, et al. Community based distribution of oral HIV selftesting kits in Zambia: a cluster-randomised trial nested in four HPTN 071 (PopART) intervention communities. Lancet HIV. 2019;6(2):e81-92.

25. Kelvin EA, Akasreku B. The evidence for HIV self-testing to increase HIV testing rates and the implementation challenges that remain. Curr HIV/AIDS Rep. 2020;17(4):281-9.

26. Mokgatle MM, Madiba S. High acceptability of HIV selftesting among technical vocational education and training college students in Gauteng and North West Province: what are the implications for the scale Up in South Africa? PLoS ONE. 2017;12(1):e0169765.

27. Kumwenda MK, Johnson CC, Choko AT, Lora W, Sibande W, Sakala D, et al. Exploring social harms during distribution of HIV self-testing kits using mixed-methods approaches in Malawi. J Int AIDS Soc. 2019;22(Suppl 1):e25251.

28. Inwani I, Chhun N, Agot K, Cleland CM, Rao SO, Nduati R, et al. Preferred HIV testing modalities among adolescent girls and young women in Kenya. J Adolesc Health. 2020;68:497-507.

29. Ziraba A, Orindi B, Muuo S, Floyd S, Birdthistle IJ, Mumah J, et al. Understanding HIV risks among adolescent girls and young women in informal settlements of Nairobi, Kenya: lessons for DREAMS. PLoS ONE. 2018;13(5):e0197479.

30. Mugo PM, Micheni M, Shangala J, Hussein MH, Graham SM, Rinke TF, et al. Uptake and acceptability of oral HIV self-testing among community pharmacy clients in Kenya: a feasibility study. PLoS ONE. 2017;12(1):e0170868.

31. National_AIDS_and_STI_Control_Programme_(NASCOP). An operational manual for the delivery of HIV Self-Testing services in Kenya. Nairobi: Ministry of Health; 2017.

32. Orasure TI. OraQuick In-home HIV test. Bethlehem: Orasure Technologies Inc; 2016.

33. Programme NASC. Framework for the Implementation of Preexposure Prophylaxis of HIV in Kenya. Nairobi, Kenya; 2017.

34. Weiner BJ, Lewis CC, Stanick C, Powell BJ, Dorsey CN, Clary AS, et al. Psychometric assessment of three newly developed implementation outcome measures. Implement Sci. 2017;12(1):108.

35. Proctor E, Silmere H, Raghavan R, Hovmand P, Aarons G, Bunger A, et al. Outcomes for implementation research: conceptual distinctions, measurement challenges, and research agenda. Adm Policy Ment Health. 2011;38(2):65-76.

36. Pantin H, Schwartz SJ, Sullivan S, Prado G, Szapocznik J. Ecodevelopmental HIV prevention programs for Hispanic adolescents. Am J Orthopsychiatry. 2004;74(4):545-58.

37. Garcia-Moreno C, Jansen HA, Ellsberg M, Heise L, Watts CH. Prevalence of intimate partner violence: findings from the WHO multi-country study on women's health and domestic violence. Lancet. 2006;368(9543):1260-9.

38. Monahan PO, Shacham E, Reece M, Kroenke K, Ong'or WO, Omollo O, et al. Validity/reliability of PHQ-9 and PHQ-2 depression scales among adults living with HIV/AIDS in western Kenya. J Gen Intern Med. 2009;24(2):189-97.

39. Bush K, Kivlahan DR, McDonell MB, Fihn SD, Bradley KA. The AUDIT alcohol consumption questions (AUDIT-C): an effective brief screening test for problem drinking. Ambulatory Care Quality Improvement Project (ACQUIP). Alcohol Use Disorders Identification Test. Arch Intern Med. 1998;158(16):1789-95.

40. Tonen-Wolyec S, Batina-Agasa S, Muwonga J, Mboumba Bouassa RS, Kayembe Tshilumba C, Belec L. Acceptability, feasibility, and individual preferences of blood-based HIV self-testing in a population-based sample of adolescents in Kisangani, Democratic Republic of the Congo. PLoS ONE. 2019;14(7):e0218795.

41. Wechsberg WM, Jewkes R, Novak SP, Kline T, Myers B, Browne FA, et al. A brief intervention for drug use, sexual risk behaviours and violence prevention with vulnerable women in South Africa: a randomised trial of the Women's Health CoOp. BMJ Open. 2013;3(5):1.

42. Cluver LD, Hodes RJ, Sherr L, Orkin FM, Meinck F, Lim AH, Ken P, et al. Social protection: potential for improving HIV outcomes among adolescents. J Int AIDS Soc. 2015;18(Suppl 6):20260.

43. Ortblad KF, Baeten JM. HIV service delivery innovation in highprevalence settings. Lancet HIV. 2020;7(6):e378-9.

44. d'Elbee M, Indravudh PP, Mwenge L, Kumwenda MM, Simwinga M, Choko AT, et al. Preferences for linkage to HIV care services 
following a reactive self-test: discrete choice experiments in Malawi and Zambia. AIDS. 2018;32(14):2043-9.

45. Kelvin EA, George G, Mwai E, Nyaga EN, Mantell JE, Romo ML, et al. Offering self-administered oral HIV testing as a choice to truck drivers in Kenya: predictors of uptake and need for guidance while self-testing. AIDS Behav. 2018;22(2):580-92.

46. Ortblad KF, Chanda MM, Musoke DK, Ngabirano T, Mwale M, Nakitende A, et al. Acceptability of HIV self-testing to support pre-exposure prophylaxis among female sex workers in Uganda and Zambia: results from two randomized controlled trials. BMC Infect Dis. 2018;18(1):503.

47. Lightfoot MA, Campbell CK, Moss N, Treves-Kagan S, Agnew E, Kang Dufour MS, et al. Using a social network strategy to distribute HIV self-test kits to African American and Latino MSM. J Acquir Immune Defic Syndr. 2018;79(1):38-45.

48. Ybarra ML, Agaba E, Chen E, Nyemara N. Iterative Development of In This toGether, the First mHealth HIV prevention program for older adolescents in Uganda. AIDS Behav. 2020;24(8):2355-68.

49. Chibanda D, Bowers T, Verhey R, Rusakaniko S, Abas M, Weiss HA, et al. The Friendship Bench programme: a cluster randomised controlled trial of a brief psychological intervention for common mental disorders delivered by lay health workers in Zimbabwe. Int J Ment Health Syst. 2015;9:21.

50. Donenberg GR, Cohen MH, Ingabire C, Fabri M, Emerson E, Kendall AD, et al. Applying the exploration preparation implementation sustainment (EPIS) framework to the Kigali Imbereheza Project for Rwandan adolescents living With HIV. J Acquir Immune Defic Syndr. 2019;82(Suppl 3):S289-98.

51. Chang W, Matambanadzo P, Takaruza A, Hatzold K, Cowan FM, Sibanda E, et al. Effect of prices, distribution strategies, and marketing on demand for HIV self-testing in Zimbabwe: a randomized clinical trial. JAMA Netw Open. 2019;2(8):e199818.

52. Daher J, Vijh R, Linthwaite B, Dave S, Kim J, Dheda K, et al. Do digital innovations for HIV and sexually transmitted infections work? Results from a systematic review (1996-2017). BMJ Open. 2017;7(11):e017604.

53. Warren CE, Mayhew SH, Hopkins J. The current status of research on the integration of sexual and reproductive health and HIV services. Stud Fam Plann. 2017;48(2):91-105.

54. Choko AT, Corbett EL, Stallard N, Maheswaran H, Lepine A, Johnson CC, et al. HIV self-testing alone or with additional interventions, including financial incentives, and linkage to care or prevention among male partners of antenatal care clinic attendees in Malawi: An adaptive multi-arm, multi-stage cluster randomised trial. PLoS Med. 2019;16(1):e1002719.

55. Moore HA, Metcalf CA, Cassidy T, Hacking D, Shroufi A, Steele SJ, et al. Investigating the addition of oral HIV self-tests among populations with high testing coverage - Do they add value? Lessons from a study in Khayelitsha, South Africa. PLoS ONE. 2019;14(5): $\mathrm{e} 0215454$.

56. Masters SH, Agot K, Obonyo B, Napierala Mavedzenge S, Maman S, Thirumurthy H. Promoting partner testing and couples testing through secondary distribution of HIV self-tests: a randomized clinical trial. PLoS Med. 2016;13(11):e1002166.

57. Simwinga M, Kumwenda MK, Dacombe RJ, Kayira L, Muzumara A, Johnson CC, et al. Ability to understand and correctly follow HIV self-test kit instructions for use: applying the cognitive interview technique in Malawi and Zambia. J Int AIDS Soc. 2019;22(Suppl 1):e25253.

58. Ortblad KF, Musoke DK, Ngabirano T, Nakitende A, Haberer JE, McConnell M, et al. Female sex workers often incorrectly interpret HIV self-test results in Uganda. J Acquir Immune Defic Syndr. 2018;79(1):e42-5.

59. Wei C, Yan L, Li J, Su X, Lippman S, Yan H. Which user errors matter during HIV self-testing? A qualitative participant observation study of men who have sex with men (MSM) in China. BMC Public Health. 2018;18(1):1108.

60. Watson V, Dacombe RJ, Williams C, Edwards T, Adams ER, Johnson CC, et al. Re-reading of OraQuick HIV-1/2 rapid antibody test results: quality assurance implications for HIV selftesting programmes. J Int AIDS Soc. 2019;22(Suppl 1):e25234.

61. Kisa R, Matovu JKB, Buregyeya E, Musoke W, Vrana-Diaz CJ, Korte JE, et al. Repeat HIV testing of individuals with discrepant HIV self-test results in Central Uganda. AIDS Res Ther. 2019;16(1):26.

Publisher's Note Springer Nature remains neutral with regard to jurisdictional claims in published maps and institutional affiliations. 